\title{
Magnetic interaction between spatially extended superconducting tunnel junctions
}

\section{Grønbech-Jensen, Niels; Samuelsen, Mogens Rugholm}

\section{Published in:}

Physical Review B Condensed Matter

Link to article, DOI:

10.1103/PhysRevB.65.144512

Publication date:

2002

Document Version

Publisher's PDF, also known as Version of record

Link back to DTU Orbit

Citation (APA):

Grønbech-Jensen, N., \& Samuelsen, M. R. (2002). Magnetic interaction between spatially extended superconducting tunnel junctions. Physical Review B Condensed Matter, 65(14), 144512.

https://doi.org/10.1103/PhysRevB.65.144512

\section{General rights}

Copyright and moral rights for the publications made accessible in the public portal are retained by the authors and/or other copyright owners and it is a condition of accessing publications that users recognise and abide by the legal requirements associated with these rights.

- Users may download and print one copy of any publication from the public portal for the purpose of private study or research.

- You may not further distribute the material or use it for any profit-making activity or commercial gain

- You may freely distribute the URL identifying the publication in the public portal

If you believe that this document breaches copyright please contact us providing details, and we will remove access to the work immediately and investigate your claim. 


\title{
Magnetic interaction between spatially extended superconducting tunnel junctions
}

\author{
Niels Grønbech-Jensen \\ Department of Applied Science, University of California, Davis, California 95616 \\ and NERSC, Lawrence Berkeley National Laboratory, Berkeley, California 94720 \\ Mogens R. Samuelsen \\ Department of Physics, The Technical University of Denmark, DK-2800 Kgs. Lyngby, Denmark
}

(Received 16 December 2001; published 29 March 2002)

\begin{abstract}
A general description of magnetic interactions between superconducting tunnel junctions is given. The description covers a wide range of possible experimental systems, and we explicitly explore two experimentally relevant limits of coupled junctions. One is the limit of junctions with tunneling distance much smaller than the London penetration depth of the superconductors, the other is the limit where the tunneling distance is much larger than the London penetration depth. The former case has previously been studied in the context of adjacent conventional Josephson junctions, while the latter has been considered through arrays of superconducting weak links based on semiconductor quantum wells with superconducting electrodes. We use the model to make direct interpretations of the published experiments and thereby propose that long-range magnetic interactions are responsible for the reported experimental signatures of coupling between tunnel junctions.
\end{abstract}

DOI: 10.1103/PhysRevB.65.144512

PACS number(s): 74.80.Dm, 74.50.+r, 85.25.Cp

\section{INTRODUCTION}

Coupling between spatially extended superconducting junctions has been investigated vigorously over the past decade. Most of this work has been focused on systems where the dominant coupling mechanism, typically inductive, is short ranged and due to the properties of common superconductors of different junctions. ${ }^{1-4}$ However, extended junctions may have spatial variations of the surface currents, thereby inducing magnetic fields in the surroundings, which in turn will cause long-range interactions between junctions. ${ }^{5,6}$ The existence of this long-range magnetic interaction was demonstrated experimentally by Holst et al.,$^{4}$ by studying phase locking between adjacent extended Josephson junctions, and, at the time, modeled by the local coupling form, ${ }^{1-3}$ which shares many characteristics with the longrange (nonlocal) coupling mechanism of Ref. 5. More recent experiments on arrays of superconducting weak links based on InAs-AlSb quantum wells with $\mathrm{Nb}$ electrodes ${ }^{7}$ may also suggest interjunction coupling due to long-range magnetic effects, and we will, therefore, investigate the nature of the external magnetic coupling in some detail.

We will initially assume that the superconductors are infinitely large in the $x$ and $y$ directions and have a thickness of $W$ in the $z$ direction. The junctions are defined by slits along the $x$ direction (parallel to the $y z$ plane, see Fig. 1). The centers of the junctions are located at $y_{i}=a_{i} \lambda_{J}$ and the electric width of the junctions is $t_{0}$ the width of the oxide layer between the superconductors. We will adopt the usual sineGordon model for the dynamics of a single junction, ${ }^{8}$

$$
\phi_{x x}-\phi_{t t}-\sin \phi=\alpha \phi_{t}-\eta,
$$

where $\phi$ represents the difference between the phases of the quantum-mechanical wave functions defining the superconducting state in each junction. In adopting this model, we are assuming that the electromagnetic dynamics in the junction is one dimensional, along the $x$ direction, and that $W \ll \lambda_{J}$. The spatial $(x, y, z)$ and temporal $(t)$ coordinates are normalized to the Josephson length $\lambda_{J}=\sqrt{\hbar / 2 e d \mu_{0} I_{c}}$ and the inverse plasma frequency $\omega_{p}^{-1}=\sqrt{\hbar \varepsilon / 2 e t_{0} I_{c}}$, respectively. The permeability is denoted by $\mu_{0}$, permittivity by $\varepsilon$, critical current density by $I_{c}$, the normalized (to $\lambda_{J}$ ) electric thickness of the junction is $b=t_{0} / \lambda_{J}$, and the magnetic thickness is $d=t_{0}+2 \lambda_{L}$, where $\lambda_{L}$ is the magnetic (London) penetration depth of the superconductors. Voltages $\phi_{t}$ are normalized to $\hbar \omega_{p} / 2 e$, the normalized surface current density of the superconductors is $-\phi_{x}$, and the characteristic energy is $H_{0}=I_{c} W \lambda_{J} \hbar / 2 e=(\hbar / 2 e)^{2} W / \mu_{0} d \lambda_{J}$. Transport of quasiparticles across the junction is given by the parameter $\alpha$ $=\rho \hbar \omega_{p} / 2 e I_{c}, \rho$ being the conductivity of the junction in the normal state. The applied bias current density $\eta$ is normalized to the critical current density $W I_{c}$.

\section{NONLOCAL MAGNETIC COUPLING}

A system of parallel superconducting junctions is sketched in Fig. 1. Following the ideas of Refs. 5 and 6 we analyze the magnetic coupling between different junctions,

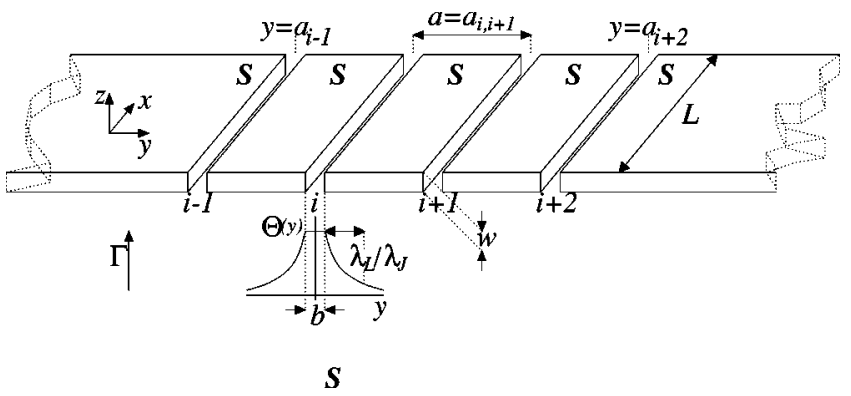

FIG. 1. Sketch of the system under consideration for $n=4$ parallel junctions. Superconductors are labeled with an $S$. All system parameters are given with the symbols of the normalized units. 
or different points along the $x$ axis within the same junction, by writing the normalized, to $\hbar / 2 e \lambda_{J}^{2}$, magnetic-flux distribution of the ith junction entering the $z>0$ half space at $(x, y)$ as

$$
m^{(i)}(x, y)=\phi_{x}^{(i)} \Theta\left(y-a_{i}\right)
$$

where the superscript $i$ identifies the junction and where

$$
\Theta(y)=\left\{\begin{array}{cc}
\frac{\lambda_{J}}{d}, & |y| \leqslant \frac{1}{2} b \\
\frac{\lambda_{J}}{d} \exp \left[-\left(|y|-\frac{b}{2}\right) \lambda_{J} / \lambda_{L}\right] & \text { otherwise. }
\end{array}\right.
$$

We here assume that the phase difference between the quantum-mechanical wave functions of the two superconductors surrounding a junction is a function of the $x$ direction only and that $\Theta$ provides the flux distribution along the $y$ direction,

$$
\int_{-\infty}^{\infty} \Theta(y) d y=1
$$

The contribution to the interaction energy from the nonlocal magnetic interaction can then be written as the integrated interaction between the magnetic-flux distribution, 5,6

$$
\begin{aligned}
H_{i n t}^{(n l)}= & \frac{1}{2} \Delta \sum_{i} \sum_{j} \iiint \int \frac{m^{(i)}\left(x_{i}, y_{i}\right) m^{(j)}\left(x_{j}^{\prime}, y_{j}^{\prime}\right)}{\sqrt{\left(x_{i}-x_{j}^{\prime}\right)^{2}+\left(y_{i}-y_{j}^{\prime}\right)^{2}}} \\
& \times d y_{i} d y_{j}^{\prime} d x_{i} d x_{j}^{\prime} \\
= & \frac{1}{2} \sum_{i} \sum_{j} \iint g_{i j}\left(x_{i}-x_{j}^{\prime}\right) \phi_{x_{i}}^{(i)} \phi_{x_{j}^{\prime}}^{(j)} d x_{i} d x_{j}^{\prime},
\end{aligned}
$$

where the kernel $g_{i j}$ is given by

$$
\begin{aligned}
g_{i j}(x) & =\Delta \iint \frac{\Theta\left(y_{i}-a_{i}\right) \Theta\left(y_{j}^{\prime}-a_{j}\right)}{\sqrt{x^{2}+\left(y_{i}-y_{j}^{\prime}\right)^{2}}} d y_{i} d y_{j}^{\prime} \\
& =\Delta \iint \frac{\Theta\left(y_{i}\right) \Theta\left(y_{j}^{\prime}\right)}{\sqrt{x^{2}+\left(y_{i}-y_{j}^{\prime}+a_{i j}\right)^{2}}} d y_{i} d y_{j}^{\prime},
\end{aligned}
$$

with $a_{i j}=a_{i}-a_{j}$. The magnitude $\Delta$ of the prefactor of the kernel $g_{i j}$ can be estimated ${ }^{5}$ in the ideal case where the superconductors extend the entire $x y$ plane,

$$
\Delta=\frac{N}{4 \pi} \frac{d}{W},
$$

where $N$ is a number, 4 or 8 , determined by the specific system geometry. One may expect the effective $N$ to be smaller than these values when finite length junctions are modeled.

The relevant Hamiltonian $H$ describing a system of magnetically coupled long Josephson junctions is then given by

$$
\begin{aligned}
H= & \sum_{i} \int\left[\frac{1}{2}\left(\phi_{x_{i}}^{(i)}\right)^{2}+\frac{1}{2}\left(\phi_{t}^{(i)}\right)^{2}+1-\cos \phi^{(i)}\right] d x_{i} \\
& +H_{\text {int }}^{(n l)}+H_{\text {int }}^{(l)},
\end{aligned}
$$

where $H_{\text {int }}^{(l)}$ is the local inductive coupling found, e.g., in Refs. 1 and 3,

$$
\begin{gathered}
H_{i n t}^{(l)}=\frac{1}{2} \sum_{i} \sum_{j} \iint g_{i j}^{(l)}\left(x_{i}-x_{j}^{\prime}\right) \phi_{x_{i}}^{(i)} \phi_{x_{j}^{\prime}}^{(j)} d x_{i} d x_{j}^{\prime}, \\
g_{i j}^{(l)}(x)=\left(\exp \left[-\frac{\lambda_{J}}{\lambda_{L}}\left|a_{i j}\right|\right]-\delta_{i j}\right) \delta(x)=\Delta_{i j}^{\prime} \delta(x),
\end{gathered}
$$

where $\delta(x)$ and $\delta_{i j}$ are Dirac's and Kronecker's delta functions, respectively.

The equation of motion for the phase of the $i$ th junction is then

$$
\begin{aligned}
\phi_{x_{i} x_{i}}^{(i)}-\phi_{t t}^{(i)}-\sin \phi^{(i)}= & \alpha_{i} \phi_{t}^{(i)}-\eta_{i}-\sum_{j} \Delta_{i j}^{\prime} \phi_{x_{j} x_{j}}^{(j)} \\
& -\sum_{j} \int g_{i j}\left(x_{i}-x_{j}^{\prime}\right) \phi_{x_{j}^{\prime} x_{j}^{\prime}}^{(j)} d x_{j}^{\prime} .
\end{aligned}
$$

We will in the following only consider the nonlocal magnetic coupling since the local coupling has already been extensively considered in the literature and since it can be directly added to the equations as indicated by the expressions above.

For systems of finite length $L \lambda_{J}, L$ being the normalized length, we will impose the usual ${ }^{8}$ boundary conditions

$$
\phi_{x_{i}}^{(i)}(0)=\phi_{x_{i}}^{(i)}(L)=\Gamma,
$$

where $\Gamma$ is the normalized external magnetic field along the $z$ direction.

\section{A. Nonlocal magnetic coupling for $d \approx 2 \lambda_{L} \gg t_{0}$}

Most Josephson junctions are characterized by this limit where $d \approx 2 \lambda_{L}$. We can here write the interaction kernel, Eq. (6), in the form

$$
g_{i j}(x)=\Delta\left(\frac{\lambda_{J}}{2 \lambda_{L}}\right)^{2} \int_{-\infty}^{\infty} \frac{\left(|\xi|+\frac{\lambda_{L}}{\lambda_{J}}\right) e^{-|\xi| \lambda_{J} / \lambda_{L}}}{\sqrt{x^{2}+\left(\xi+a_{i j}\right)^{2}}} d \xi .
$$

For $i=j$, we can express the kernel exactly as ${ }^{9}$

$$
\begin{aligned}
g_{i i}(x)= & \Delta \frac{\pi \lambda_{J}}{4 \lambda_{L}}\left\{\mathcal{H}_{0}\left(\frac{\lambda_{J}}{\lambda_{L}}|x|\right)-\mathcal{N}_{0}\left(\frac{\lambda_{J}}{\lambda_{L}}|x|\right)\right. \\
& \left.+\frac{\lambda_{J}}{\lambda_{L}}|x|\left[\mathcal{H}_{1}\left(\frac{\lambda_{J}}{\lambda_{L}}|x|\right)-\mathcal{N}_{1}\left(\frac{\lambda_{J}}{\lambda_{L}}|x|\right)-\frac{2}{\pi}\right]\right\},
\end{aligned}
$$


where $\mathcal{H}_{n}$ and $\mathcal{N}_{n}$ are the $n$th order Struve ${ }^{10}$ and Weber ${ }^{11}$ functions, respectively.

This expression has the two distinct limiting forms

$$
\begin{aligned}
g_{i i}(x) \rightarrow g_{i i}^{(\infty)}(x)=\frac{\Delta}{\sqrt{x^{2}+\left(2 \frac{\lambda_{L}}{\lambda_{J}}\right)^{2}}} \text { for }|x| \gg \frac{\lambda_{L}}{\lambda_{J}}, \\
g_{i i}(x) \rightarrow g_{i i}^{(0)}(x) \\
=\frac{\Delta}{2} \frac{\lambda_{J}}{\lambda_{L}}\left[1-\mathcal{C}+\ln \left(2 \frac{\lambda_{L}}{\lambda_{J}}\right)-\ln |x|\right] \text { for }|x| \ll \frac{\lambda_{L}}{\lambda_{J}},
\end{aligned}
$$

where the first expression has a leading error term $\propto\left(x \lambda_{J} / \lambda_{L}\right)^{-5}$. $\mathcal{C}$ is the Euler constant. ${ }^{12}$

For $i \neq j$, we cannot express the kernel $g_{i j}(x)$ explicitly. However, one can, for large $|x| \lambda_{J} / \lambda_{L}$, approximate the kernel with

$$
g_{i j}(x) \rightarrow g_{i j}^{(\infty)}(x)=\frac{\Delta}{\sqrt{x^{2}+\left(2 \frac{\lambda_{L}}{\lambda_{J}}\right)^{2}+a_{i j}^{2}}} \text { for }|x| \gg \frac{\lambda_{L}}{\lambda_{J}},
$$

with the leading error term being $\propto\left(x \lambda_{J} / \lambda_{L}\right)^{-5}$. Since the magnetic penetration depth $\lambda_{L}$ is usually orders of magnitude smaller than the characteristic length scale $\lambda_{J}$ at which $\phi^{(i)}\left(x_{i}\right)$ varies in Josephson junctions, we can with very good approximation use the above limiting expressions, $g_{i j}^{(\infty)}(x)$ for $x \neq 0$.

The remaining contribution for $|x|<\lambda_{L} / \lambda_{J}$ can thus be accounted for through a purely local interaction of the form

$$
\begin{aligned}
g_{i j}^{(0)}(x) & =\left(\int_{-\infty}^{\infty}\left[g_{i j}(\xi)-g_{i j}^{(\infty)}(\xi)\right] d \xi\right) \delta(x) \\
& =\Delta \delta(x)\left\{\begin{array}{cl}
\ln 4+2 \mathcal{C}-1 \approx 1.541 & \text { for } \quad a_{i j}=0 \\
\frac{8}{a_{i j}^{2}} \frac{\lambda_{L}^{2}}{\lambda_{J}^{2}} & \text { for } \quad\left|a_{i j}\right| \gg \frac{\lambda_{L}}{\lambda_{J}} .
\end{array}\right.
\end{aligned}
$$

For systems where $\lambda_{L} / \lambda_{J} \ll 1$ and $\left|a_{i}-a_{i \pm 1}\right| \gg \lambda_{L} / \lambda_{J}$, we can, therefore, with good approximation write

$$
g_{i j}(x) \approx g_{i j}^{(0)}(x)+g_{i j}^{(\infty)}(x)
$$

for all $i, j$.

\section{B. Nonlocal magnetic coupling for $\lambda_{L} \ll t_{0} \approx d$}

While this limit of system parameters is not usually relevant for Josephson systems, experiments on superlattices of semiconductor quantum wells and superconducting electrodes ${ }^{7}$ indicate that tunneling between superconductors can be facilitated over distances of $500 \mathrm{~nm}$, and, thus, that a
Josephson effect can be expected in systems where the tunneling distance $t_{0}$ is much larger than the magnetic penetration depth $\lambda_{L}$. We will, therefore, analyze the long-range magnetic interaction in this limit, providing a model for the interaction between periodic arrays of semiconductor weak links and superconducting lines.

The interaction kernel, Eq. (6), is here given by

$$
\begin{aligned}
g_{i j}(x) & =\Delta\left(\frac{\lambda_{J}}{d}\right)^{2} \int_{-b / 2}^{b / 2} \int_{-b / 2}^{b / 2} \frac{1}{\sqrt{x^{2}+\left(y_{i}-y_{j}+a_{i j}\right)^{2}}} d y_{i} d y_{j} \\
& =\frac{\Delta}{b^{2}} \int_{-b}^{b} \frac{1-|\xi|}{\sqrt{x^{2}+\left(\xi+a_{i j}\right)^{2}}} d \xi .
\end{aligned}
$$

This expression can be written in the exact form

$$
\begin{aligned}
g_{i j}(x)= & \frac{\Delta}{b^{2}}\left[2 \sqrt{x^{2}+a_{i j}^{2}}-\sqrt{x^{2}+\left(a_{i j}+b\right)^{2}}-\sqrt{x^{2}+\left(a_{i j}-b\right)^{2}}\right. \\
& +\left(a_{i j}+b\right) \ln \frac{\sqrt{x^{2}+\left(a_{i j}+b\right)^{2}}+a_{i j}+b}{\sqrt{x^{2}+a_{i j}^{2}}+a_{i j}} \\
& \left.-\left(a_{i j}-b\right) \ln \frac{\sqrt{x^{2}+\left(a_{i j}-b\right)^{2}}-\left(a_{i j}-b\right)}{\sqrt{x^{2}+a_{i j}^{2}}-a_{i j}}\right]
\end{aligned}
$$

It is here important to recall that $\left|a_{i j}\right|>b$ for $i \neq j$. For $a_{i j}$ $=0(i=j)$ Eq. (22) reads

$$
g_{i i}(x)=2 \frac{\Delta}{b^{2}}\left[|x|-\sqrt{x^{2}+b^{2}}+b \ln \frac{b+\sqrt{x^{2}+b^{2}}}{|x|}\right]
$$

and the asymptotic form of the general expression for large $|x|$ is

$$
g_{i j}(x) \rightarrow g_{i j}^{(\infty)}(x)=\frac{\Delta}{\sqrt{x^{2}+a_{i j}^{2}+\frac{1}{6} b^{2}}} \text { for }|x| \gg \sqrt{a_{i j}^{2}+b^{2}},
$$

with leading error term $\propto x^{-5}$.

Equation (22) is exact, but still poses a few concerns for $x=0$. However, $g_{i i}(x)$ has only a logarithmic singularity, which is well behaved when integrated, and $g_{i j}(x)$ has the well-defined limiting value

$$
\begin{aligned}
g_{i j}(0)= & \frac{\Delta}{b} \frac{\left|a_{i j}\right|}{b}\left[\left(1+\frac{b}{\left|a_{i j}\right|}\right) \ln \left(1+\frac{b}{\left|a_{i j}\right|}\right)\right. \\
& \left.+\left(1-\frac{b}{\left|a_{i j}\right|}\right) \ln \left(1-\frac{b}{\left|a_{i j}\right|}\right)\right] \text { for } i \neq j .
\end{aligned}
$$

Assuming that the magnetic-flux distribution can be expressed by Eq. (2), we have then provided the kernel that determines the magnetic interaction between tunnel junctions 
in the limit where the the magnetic and electric thickness of the junctions are equal, $\lambda_{L} \ll t_{0} \approx d$.

\section{NUMERICAL SIMULATIONS}

In order to explore the possible relevance of the above coupling mechanism, we have performed numerical simulations of experimentally relevant superconducting systems, which produce measurements that may be interpreted in light of nonlocal magnetic coupling. The following two sections consider simulations of equations of motion in the form of Eq. (11), where the interaction kernel $g_{i j}$ takes the form of the above two extreme parameter limits. We are not considering the local inductive coupling $\Delta_{i j}^{\prime}$.

\section{A. Nonlocal magnetic coupling for $d \approx 2 \lambda_{L} \gg t_{0}$}

Observation of phase locking between two (or more) adjacent extended Josephson junctions have been reported in cases where the oscillators are operated in self-resonant (fluxon) modes. ${ }^{4,13,14}$ The experiments reported in Refs. 4 were performed on a system with two junctions of length $L$ $\approx 4$, width $w \approx 0.2$, interjunction distance $a \approx 0.35,0.75$, and magnetic thickness $d \approx 0.0009 \lambda_{J}$; all lengths are normalized to $\lambda_{J} \approx 100 \mu \mathrm{m}$. Phase locking between fluxon modes was reported in this system both when the bias currents of the two junctions were of the same $(\sigma=1)$ and opposite $(\sigma=$ -1 ) sign and a local coupling model was adopted to explain the core features of the experimental data. We will here demonstrate the phase-locking results based on the nonlocal magnetic coupling, whose strength and functional form is almost entirely given by the geometry of the system.

The above geometry provides for an interjunction coupling parameter [see Eq. (7)]

$$
\Delta \approx 0.00145 \text {, }
$$

where we have used $N=4$ since the junctions are adjacent, and a coupling parameter of

$$
\Delta \approx 0.00290
$$

for the junction self-interaction $\left[g_{i i}(x)\right]$. We have performed the presented simulations with a damping coefficient of $\alpha$ $=0.05$ and external magnetic field $\Gamma=0$. The experimental system consists of two junctions with slightly different system parameters, and the study of phase locking between fluxon oscillations in the different junctions was conducted by having two independent current sources biasing the junctions. We will, for simplicity, model the junctions as being identical except for their individual bias currents.

The results of the numerical simulations are summarized in Figs. 2 and 3. Figure 2 shows the simulated dc-average current-voltage characteristics of the two junctions, both operated in a zero-field-step (ZFS) mode (single oscillating fluxon), with identical magnitude bias current $\left(\eta_{0}=\eta_{1}\right.$ $\left.=\sigma \eta_{2}, \sigma= \pm 1\right)$. Given the small magnitude(s) of $\Delta$, we find that the current-voltage characteristics are almost independent of the current polarity $\sigma= \pm 1$. However, due to the self-coupling, given by $g_{i i}(x)$, the ZFS's are not stable for all bias currents up to the critical currents, $\left|\eta_{i}\right|=1$, of the junc-

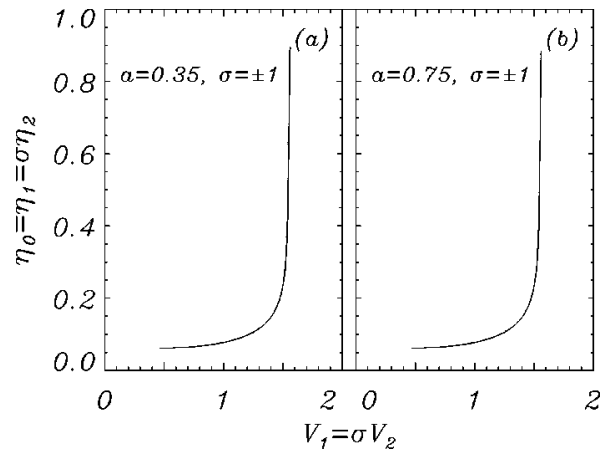

FIG. 2. Dc current-voltage charateristics $\left(\eta_{0}, V_{i}\right)$ for $n=2$ identical adjacent Josephson junctions with parameters $L=4, \alpha$ $=0.05, \lambda_{L} / \lambda_{J}=0.0009$, and (a) $a=0.35$, (b) $a=0.75$, operated at the first zero-field step for $\sigma= \pm 1$.

tions. Figure 3 shows how the range in bias current difference, $\Delta \eta=\max \left|\eta_{1}-\sigma \eta_{2}\right|$, for which the average voltages of the junctions are identical $\left(\left\langle V_{1}\right\rangle=\sigma\left\langle V_{2}\right\rangle\right)$ as a function of the bias point $\eta_{0}=\frac{1}{2}\left(\eta_{1}+\sigma \eta_{2}\right)$. The simulation results clearly show that nonzero, and measurable, phase-locking ranges may be expected as a result of magnetic interactions. We further observe that the locking range in bias current is roughly independent of the bias polarity $\sigma$ and that the locking ranges for the interjunction distance $a=0.35$ are roughly four times those the locking ranges of the system with $a$ $=0.75$, in reasonable agreement with the experimental data. ${ }^{4}$ Making quantitative comparisons between our simulation data and the corresponding experiments, we notice that the simulations exhibit maximum locking ranges of about the size exhibited by the experiments. Some discrepancy is of course not unexpected, since the experimental system is reported with, e.g., slightly different critical currents of the junctions, a large uncertainty in the characteristic Josephson length, etc. Additionally, the above theory is based on ideal geometries where considerations of magnetic interactions can be simplified (e.g., the parameter $N$ in the magnitude $\Delta$ of the interaction kernel is likely smaller than predicted, as mentioned above). With such considerations in mind, the agreement between the simulated theory and the experimental data is remarkably good and demonstrates the potential

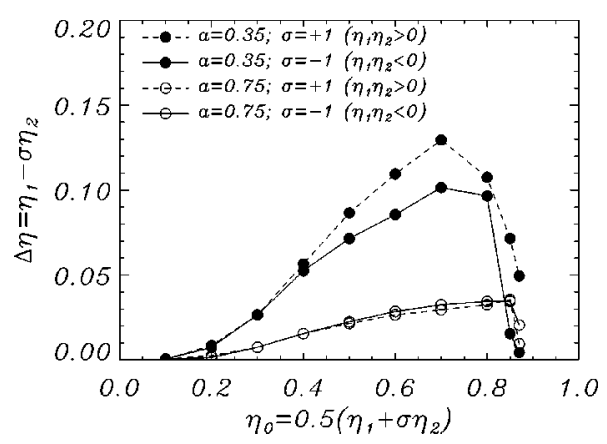

FIG. 3. Ranges of phase locking, $\Delta \eta=\max \left|\eta_{1}-\sigma \eta_{2}\right|$, as a function of the current bias point $\eta_{0}=\frac{1}{2}\left(\eta_{1}+\sigma \eta_{2}\right)$ for the junctions whose current-voltage characteristics are shown in Fig. 2. The junctions are biased at the first zero-field step shown in Fig. 2. 
importance of the long-range magnetic interaction and cross talk between extended Josephson junctions.

\section{B. Nonlocal magnetic coupling for $\lambda_{L} \ll t_{0} \approx d$}

Current-voltage measurements on periodic superlattices of gratings of superconducting $\mathrm{Nb}$ electrodes and InAs-AlSb quantum wells ${ }^{7}$ in a weak magnetic field have revealed resistance with periodic components as a function of the applied magnetic field. The oscillation period has been found to correspond to a flux quantum being injected into (ejected from) the grating cells. However, for long grating cells a doubling of the frequency is observed for low applied magnetic fields. This frequency doubling was interpreted as a result of symmetry breaking in the flux configurations of the superconducting gratings.

We will here investigate this interpretation using our longrange magnetic coupling as the coupling mechanism between the superconducting gratings. Since the superlattice of gratings exhibits periodic resistivity behavior as a function of magnetic field, and since a critical current is measured across the superlattice, we assume that Josephson coupling exists between the superconducting $\mathrm{Nb}$ strips. However, since the physical (tunneling) distance between the superconductors is $b \lambda_{J} \approx 500 \mathrm{~nm} \gg \lambda_{L} \approx 45 \mathrm{~nm}$, we will consider the magnetic coupling of Sec. IIB. The Josephson penetration depth of each extended weak link is estimated to be $\lambda_{J} \approx 2.3 \mu \mathrm{m}$ from the experiments. Thus, all the relevant geometric parameters, $\quad a=960 \mathrm{~nm} / \lambda_{J}, \quad b, \quad L \leqslant 95 \mu \mathrm{m} / \lambda_{J}$, and $w$ $=15 \mathrm{~nm} / \lambda_{J}$, can be appropriately normalized. The critical current density of the lattice is measured to be $W I_{c}$ $\approx 1.26 \mathrm{~A} / \mathrm{m}$.

We have conducted numerical simulations of systems consisting of up to $n=18$ overdamped weak links with the above system parameters and with normalized lengths of $L$ $=10-30$ for varying normalized applied magnetic fields $\Gamma$. Since the experiments are probing a dynamical resistance at a low frequency of $497 \mathrm{~Hz}$, we cannot expect to simulate the exact measurement within such a long time scale. Instead, we have decided to numerically measure the relative magnetization $\Delta M_{i}=\phi^{(i)}(L)-\phi^{(i)}(0)-L \Gamma$ for each junction in the applied magnetic field, without bias current. Thus, we apply the desired magnetic field and let the system relax until $\dot{\phi}^{(i)}(x)=0$ for all $x$ and $i$, whereafter the relative magnetization is measured. We then define the total relative magnetization $\Delta M=\left\langle\Delta M_{i}\right\rangle_{i}$ as a measurement relevant for the experimentally observed dynamical resistance.

Figure 4 shows the magnetization simulation results for the above parameters with $L=10$ and $L=20$ as a function of the (decreasing) applied magnetic field (normalized to integer number of flux quanta per junction). The results clearly show that the system responds periodically with the magnetic field and that the period is the flux quantum. Both simulated lengths show that the periodic response vanishes at very low magnetic fields. However, for the longer system, $L=20$, we observe a transition into frequency doubling at intermediate magnetic fields-all these observations are in direct agreement with the experimental observations of the dynamical resistance as a function of magnetic field. ${ }^{7}$ In or-

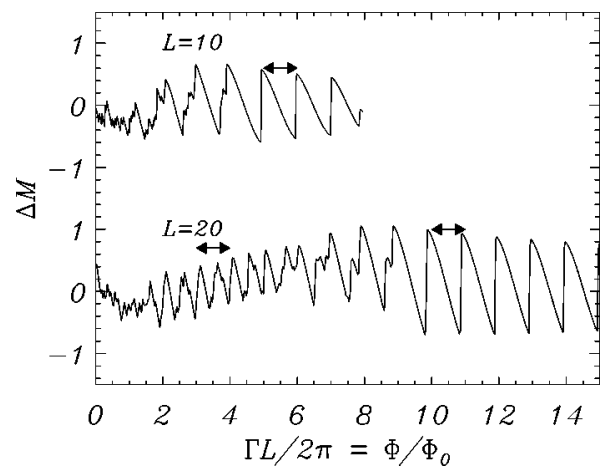

FIG. 4. Normalized relative magnetization $\Delta M=\left\langle\phi^{(i)}(L)\right.$ $\left.-\phi^{(i)}(0)\right\rangle_{i}-L \Gamma$ as a function of normalized applied magnetic field $\Gamma$ for systems of $n=18$ junctions with $\left(b \gg \lambda_{L} / \lambda_{J}\right)$ coupling parameters $a=0.42, b=0.22$, and $\Delta=0.3$. The horizontal axis is scaled to the flux quantum, $\Phi_{0}=h / 2 e$. Indicated by the arrows $(\leftrightarrow)$ of unit length, large applied magnetic fields $\Gamma$ result in magnetization period of $\Phi_{0}$, while the longer system $(L=20)$ exhibits a frequency doubling for smaller values of $\Gamma$.

der to investigate the more detailed reason for this behavior, we have, in Fig. 5, displayed the individual relative magnetizations $\Delta M_{i}$ for $i=5-14$ and $L=20$ as a function of the magnetic field. For large fields we observe that all junctions behave identically in their magnetizations (the vertical dashed lines define field intervals of a single flux quantum

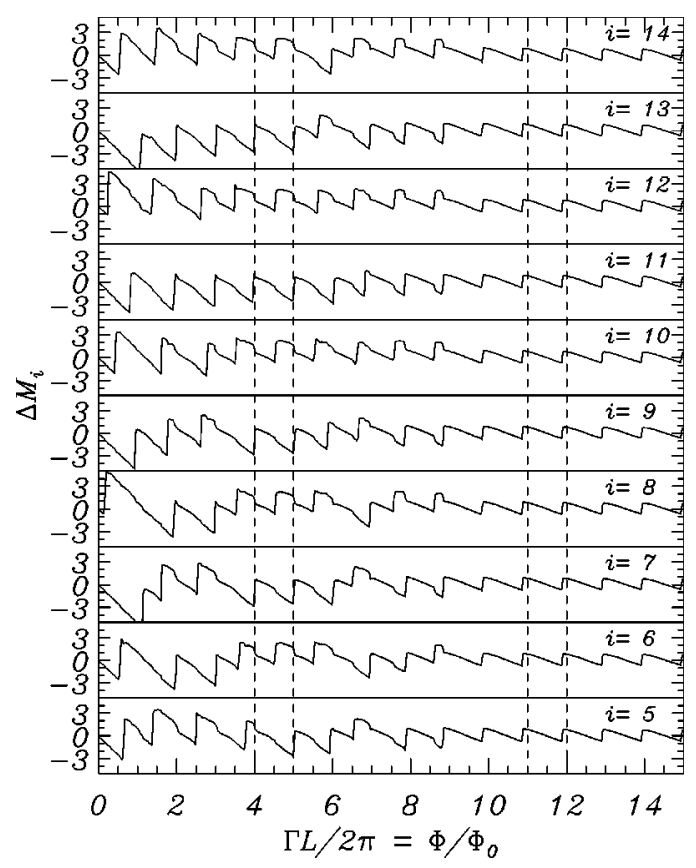

FIG. 5. Normalized relative magnetization $\Delta M_{i}=\left(\phi^{(i)}(L)\right.$ $\left.-\phi^{(i)}(0)\right)-L \Gamma$ of junctions $5-14$ of the $n=18$ participating junctions of length $L=20$ described in Fig. 2. $\Delta M_{i}$ is shown as a function of normalized applied magnetic field $\Gamma$. Vertical sections indicated by dashed lines demonstrate that the global periodicity $(\Delta M$ $\left.=\left\langle\Delta M_{i}\right\rangle_{i}\right)$ seen in Fig. 2 is due to "in-phase" $\left(\phi_{x_{i}}^{(i)}=\phi_{x_{i \pm 1}}^{(i \pm 1)}\right)$ magnetic-field distribution in the junctions for high $\Gamma$, and "out-ofphase" $\left(\phi_{x_{i}}^{(i)} \neq \phi_{x_{i \pm 1}}^{(i \pm 1)}\right)$ magnetic-field distribution for low external fields. 
per junction). However, as the field is decreased, the flux distribution of neighboring junctions slide out of phase resulting in a frequency doubling of the total magnetization. This scenario corresponds exactly to the suggested explanation given by Thomas et al. in Ref. 7 and it can be justified through the following intuition. All the junctions will respond identically (in phase) if no magnetic interaction between the junctions is present. Thus, when the external field is strong, the boundary effects dominate the flux structure. Additionally, at a dense packing of fluxons, the effective repulsive flux interaction between junctions is vanishing. However, since unipolar fluxons are mutually repulsive, decreasing magnetic fields result in the boundary effect eventually becoming insignificant compared to the internal repulsion between the flux modes, which will favor an out-of-phase flux distribution between junctions. Since the effective repulsion between the fluxon modes of the different junctions depends strongly on the length of the junctions [see, e.g., Eq. (5)] and since the boundary effect is independent of the system length, the frequency doubling transition may not be observed in the shorter junction systems since the boundary effects become relatively more important for shorter systems.

As we did for the above case of coupled long Josephson junctions, we conclude here that the long-range magnetic coupling model has provided a strong component to the interpretation of the experimental data of the behavior of superlattices of superconducting weak links in the limit of $d$ $\approx t_{0}$. While the simulations do not directly account for the low frequency dynamical measurements, and while we simulate only $n=18$ junctions instead of the experimental 310 , we submit that the agreement between simulation and experimental observation is quite good considering the simplicity of the model.

\section{CONCLUSION}

Based on the above simple theory and the accompanying simulations with their direct correspondence with published experiments, we conclude that the long-range magnetic interaction is relevant for a wide variety of superconducting systems where the dynamics and configurations of flux quanta are present. We have demonstrated the importance through two very different experimental situations and interpreted the experimentally observed features as originating from the magnetic coupling. It is important to emphasize that while the agreement between our simulation results and the published experimental observations are quite reasonable, the strengths and form of coupling used in the simulations arise directly from the experimental system parameters without fitting. Thus, even though specific quantitative agreement with experimental data may depend on system details, such as the electromagnetic properties of the surroundings, the principles of the simple magnetic model will provide a good starting point for interpreting many features of experimentally observed coupling between fluxon behavior.

\section{ACKNOWLEDGMENTS}

We acknowledge many useful discussions with J. A. Blackburn and M. Cirillo regarding experiments on coupled Josephson junctions and with A. R. Bishop, H. Kroemer, and D. Scalapino during the initial parts of this work regarding the semiconductor-superconductor superlattices. This work was supported in part by the Director, Office of Advanced Scientific Computing Research, Division of Mathematical, Information, and Computational Sciences of the U.S. Department of Energy under Contract No. DE-AC03-76SF00098.

${ }^{1}$ K.L. Ngai, Phys. Rev. 182, 555 (1969).

${ }^{2}$ M.B. Mineev, G.S. Mkrtchyan, and V.V. Schmidt, J. Low Temp. Phys. 45, 497 (1981).

${ }^{3}$ A.F. Volkov, Pis'ma Zh. Eksp. Teor. Fiz. 45, 299 (1987) [JETP Lett. 45, 376 (1987)].

${ }^{4}$ T. Holst, J.B. Hansen, N. Grønbech-Jensen, and J.A. Blackburn, Phys. Rev. B 42, 127 (1990); IEEE Trans. Magn. 27, 2704 (1991).

${ }^{5}$ N. Grønbech-Jensen and M.R. Samuelsen, Phys. Rev. Lett. 74, 170 (1995).

${ }^{6}$ Yu.M. Ivanchenko, Phys. Rev. B 52, 79 (1995).

${ }^{7}$ M. Thomas, H.R. Blank, K.C. Wong, C. Nguyen, H. Kroemer, and E.L. Hu, Phys. Rev. B 54, 2311 (1996).

${ }^{8}$ See, e.g., A. Barone and G. Paternó, Physics and Applications of the Josephson Effect (Wiley, New York, 1982).

${ }^{9}$ The kernel $g_{i i}(x)$ was previously derived (Ref. 6), but the result only included the terms with zeroth-order Struve and Weber functions.

${ }^{10}$ See, e.g., Handbook of Mathematical Functions, edited by R. M. Abramowitz and I.E. Stegun, Applied Mathematics Series Vol. 55 (National Bureau of Standards, Washington, D.C., 1964), p. 496.

${ }^{11}$ See, e.g., Handbook of Mathematical Functions (Ref. 10), p. 358. It is also called the Bessel function of the second kind or the Neumann function according to I.S. Gradshteyn and I.M. Ryzhik, Table of Integrals, Series, and Products, 5th ed. (Academic Press, San Diego, 1994), p. 960.

${ }^{12}$ See, e.g., Ref. 10.

${ }^{13}$ T.F. Finnegan and S. Wahlsten, Appl. Phys. Lett. 21, 541 (1972).

${ }^{14}$ M. Cirillo and F.L. Lloyd, J. Appl. Phys. 61, 2581 (1987). 